\title{
Eficacia del monepantel para el control de aislamientos de Haemonchus contortus y Trichostrongylus spp. con resistencia múltiple (ivermectina y febendazole) en caprinos
}

\author{
Cooper $\mathrm{L}^{1}$, Cerutti $\mathrm{J}^{1}$, Mohn $\mathrm{C}^{2}$, Torrents $\mathrm{J}^{3}$, Suarez Archilla $\mathrm{G}^{4}$, Anziani OS $\mathrm{S}^{1,4^{*}}$ \\ 1 Facultad de Ciencias Agropecuarias Universidad Católica de Córdoba, Córdoba, Argentina \\ AER INTA Deán Funes, Córdoba, Argentina \\ Facultad de Ciencias Veterinarias, Universidad Nacional del Litoral, Santa Fe, Argentina \\ EEA INTA Rafaela, Santa Fe, Argentina \\ * Correspondencia: O.S. Anziani, EEA INTA Rafaela, CC 22, 2300 Rafaela, Santa Fe, Argentina. \\ E-mail: anziani.oscar@inta.gob.ar
}

Recibido: 7 Julio 2016. Aceptado: 22 Agosto 2016. Disponible en línea: 24 Agosto 2016

Editor: P. Beldomenico

\begin{abstract}
RESUMEN. El monepantel es un nuevo antihelmíntico registrado en nuestro país exclusivamente para el control de los nematodes gastrointestinales de los ovinos y su uso ha sido direccionado mayormente hacia el control de parásitos resistentes a las clases de antihelmínticos disponibles actualmente. Estos mismos nematodes también parasitan a los caprinos, pero en estos rumiantes la patofisiología y la respuesta a los antihelmínticos es diferente, lo cual resulta en un mayor parasitismo y complejidad en el manejo de la resistencia. La presente comunicación informa sobre la eficacia del monepantel en dos hatos caprinos mantenidos bajo condiciones de campo y parasitados naturalmente por los géneros de nematodes gastrointestinales más comunes del área central de la Argentina (Haemonchus y Trichostrongylus) y portando alelos de resistencia múltiple (ivermectina y febendazole). El test de reducción en el conteo de huevos post tratamiento comparando diversas fórmulas, indicaron que en todos los hatos el monepantel por vía oral y a la dosis de $3,75 \mathrm{mg} / \mathrm{kg}$ de peso (1,5 veces mayor a la dosis ovina), resultó en eficacias del $99 \%$ al $100 \%$. Se realizan breves consideraciones sobre el uso potencial de esta droga en caprinos.
\end{abstract}

SUMMARY. Monepantel effectiveness against Haemonchus contortus and Trichostrongylus spp. with multiple resistance (ivermectin and febendazole) in goats. Monepantel is a new anthelmintic registered in Argentina exclusively for control of gastrointestinal nematodes of sheep and mostly directed toward controlling resistant parasites to current available classes of anthelmintics. The same nematodes also parasitize goats but pathophysiology and response are different in these hosts resulting in more severe parasitism and complexity in handling anthelmintic resistance. This report assess the efficacy of monepantel in goats maintained under field conditions and naturally parasitized by most common gastrointestinal nematodes from central Argentina (Haemonchus and Trichostrongylus) carrying alleles of multiple resistance (ivermectin and febendazole). According to the egg count reduction test, efficacies of $99 \%$ to $100 \%$ were observed after monepantel treatment at $3.75 \mathrm{mg} / \mathrm{kg}$ orally (1.5 ovine dose).

Palabras clave: resistencia antihelmíntica; caprinos; monepantel; Argentina

Key words: anthelmintic resistance; goats; monepantel; Argentina

En los ovinos y caprinos, los nematodes gastrointestinales constituyen, desde una perspectiva global, el principal problema sanitario y productivo (Waller, 2003; Kaplan, 2004; Bessier, 2007). Ambas especies de rumiantes comparten los mismos géneros parasitarios de nematodes gastrointestinales, pero la formación de la respuesta inmune así como la expresión de la misma es menos eficiente en caprinos que en ovinos, resultando en un mayor parasitismo en la primera de estas especies (Hoste et al., 2010). Asimismo, en los caprinos la absorción de los antihelmínticos es menor que en otros rumiantes y también metabolizan y eliminan estas drogas en forma más rápida (Lespine et al., 2012).

La resistencia antihelmíntica está comprometiendo seriamente la sustentabilidad del control de estos nematodes en ambas especies de rumiantes lo cual resulta en severas pérdidas económicas y en la dispersión de parásitos portando alelos con resistencia simultánea a drogas con distinto modo de acción (Romero et al., 2007; Romero et al., 2013; Anziani y Muchiut ,2014; Anziani y Fiel, 2015). El monepantel es un nuevo antihelmíntico desarrollado para ovinos 
fundamentalmente para el control de aislamientos con resistencia múltiple (Kaminsky et al., 2009; Steffan et al., 2011); y en nuestro país, así como en la mayoría del mundo donde se comercializa, sus indicaciones de uso son exclusivamente restringidas a los ovinos.

El objetivo de la presente comunicación es informar sobre la actividad del monepantel en caprinos utilizando un test de reducción en el conteo de huevos (TRCH) en dos hatos naturalmente parasitados con aislamientos de Haemonchus contortus y Trichostrongylus spp. con resistencia múltiple (febendazol e ivermectina).

\section{Localización de las experiencias, antecedentes y} animales utilizados

Las experiencias se llevaron a cabo entre Diciembre de 2015 y Mayo de 2016 en dos hatos caprinos pertenecientes a la AER INTA Deán Funes y a la Universidad Católica de Córdoba. En la primera se realizaron dos experiencias (A y B) utilizando 34 y 28 animales de raza Nubian, mientras que en la Universidad Católica de Córdoba (experiencia C) se utilizaron 14 cabras mestizas. Todas las experiencias contemplaron el uso de animales de diferentes edades y de ambos sexos. En ambos establecimientos existían antecedentes documentados de nematodes gastrointestinales (Haemonchus contortus y Trichostrongylus spp.) con resistencia simultánea a ivermectina y febendazol (Anziani et al., 2010).

Diseño de la experiencia, tratamientos y determinaciones parasitológicas

La eficacia de la droga se determinó a través del test de reducción en el conteo de huevos (TRCH). Entre los días -1 y 0 de cada una de las experiencias, los animales fueron pesados, identificados con caravanas y se tomaron muestras de materia fecal para determinar el número de huevos de nematodes por gramo de heces $(h p g)$ mediante la técnica de Mc Master modificada (Roberts y O' Sullivan, 1949). Se seleccionaron para ambas experiencias animales con valores del $h p g \geq 250$. Alícuotas de materia fecal fueron obtenidas para la realización de coprocultivos y recuperación de larvas de tercer estadio a través de un aparato de Baermann para identificación de los géneros de nematodes intervinientes. En el día 0 se realizaron los tratamientos con monepantel por vía oral a la dosis de $3,75 \mathrm{mg} / \mathrm{kg}$ de peso vivo (equivalente a 1,5 veces la dosis indicada en ovinos) y entre los días 10 y 12 posteriores al tratamiento se tomaron nuevas muestras de materia fecal para establecer el TRCH. En dos de las experiencias ( $\mathrm{B}$ y $\mathrm{C}$ ) y para este mismo período se determinó además la evolución del volumen globular por la técnica del microhematocrito (ht).

Análisis de datos y criterios para la evaluación de susceptibilidad y/o resistencia
Para el TRCH se siguieron las recomendaciones generales de la World Association of Veterinary Parasitology (Coles et al., 1992). En las tres experiencias se compararon los valores del $h p g$ pre y pos-tratamiento de los mismos animales utilizando las siguientes fórmulas:

1) $\quad \mathrm{TRCH}=100 \times(1-[\mathrm{T} 2 / \mathrm{T} 1])$ donde $\mathrm{T} 2$ es el promedio de los valores de $h p g$ pos-tratamiento y $\mathrm{T} 1$ el promedio de los valores de $h p g$ pre-tratamiento (McKenna, 2006).

2) $\operatorname{TRCH}=100 \times(1-p)$ donde $p=x / n$, siendo " $x$ " la sumatoria de los huevos pos-tratamiento $y$ " $\mathrm{n}$ " la sumatoria de los huevos pre-tratamiento (Dobson et al., 2012).

3) $\mathrm{TRCH}=(1 / \mathrm{n}) \times \Sigma(100 \times(1-\mathrm{Ti} 2 / \mathrm{Ti} 1))$, donde los valores de $\mathrm{Ti}$ son los valores de $h p g$ individuales, realizando una reducción individual. Esta fórmula está basada en el promedio de la sumatoria de las reducciones individuales (Cabaret, 2004).

Los valores del $h t$ pre y post tratamientos se analizaron utilizando el test de Student, InfoStat (Universidad Nacional de Córdoba, www.InfoStat.com.ar).

En las experiencias A y B, la clasificación taxonómica de las larvas obtenidas en los coprocultivos pretratamiento indicó la presencia casi exclusiva de Haemonchus contortus (98\%) y el resto perteneciente al género Trichostrongylus spp., mientras que en la experiencia $\mathrm{C}$ la participación relativa de estos géneros fue de $78 \%$ y $22 \%$, respectivamente. Debido a que existen algunas evidencias conflictivas sobre que fórmula utilizar para determinar la eficacia de los antihelmínticos cuando se utiliza el TRCH (Falzon et al., 2015), en la presente experiencia y para asegurar la interpretación de los resultados se utilizaron tres fórmulas distintas, no observándose diferencias significativas entre las mismas. Los tratamientos resultaron en reducciones del $h p g$ utilizando las diferentes fórmulas que variaron entre el $99,7 \%$ y $100 \%$ para las tres experiencias. Una síntesis de los resultados sobre la eficacia de esta droga es presentada en la Tabla 1.

Estos valores de eficacia evaluados por el TRCH concuerdan con estudios sobre conteos de nematodes adultos de diferentes géneros en infecciones naturales y artificiales informadas en cabras de Nueva Zelanda y Australia utilizando la misma dosis antihelmíntica (Rolfe et al., 2011). En nuestra experiencia, la droga utilizada a la dosis de $3,75 \mathrm{mg} / \mathrm{kg}$ (1,5 veces mayor a la dosis ovina) resultó altamente efectiva contra Haemonchus spp. y Trichostrongylus spp. portando alelos resistentes a la ivermectina y el febendazol. El primero de estos géneros es indudablemente el de mayor importancia para los caprinos del área central de la Argentina y la resistencia hacia ambos antihelmínticos citadas anteriormente es extremadamente común (Anziani et al., 2010; Aguirre y Cafrune, 2013). La mayor respuesta en el aumento del $h t$ observada en la 
Tabla 1: Eficacia de monepantel (3,75 mg/kg oral) sobre aislamientos de Haemonchus contortus y Trichostrongylus spp. con resistencia múltiple en caprinos naturalmente parasitados. Promedios de los hpg (rango) y porcentaje en el TRCH comparando diferentes fórmulas. Fórmula 1 (McKenna, 2006); Fórmula 2 (Dobson et al, 2012); Fórmula 3 (Cabaret, 2004).

\begin{tabular}{|c|c|c|c|c|c|c|c|c|}
\hline \multirow[b]{2}{*}{ Experiencia } & \multirow[b]{2}{*}{$\begin{array}{c}\text { № de } \\
\text { animales }\end{array}$} & \multicolumn{2}{|c|}{$\begin{array}{l}\text { Promedio hpg } \\
\text { (rango) }\end{array}$} & \multicolumn{3}{|c|}{ TRCH (\%) } & \multicolumn{2}{|c|}{$\begin{array}{l}\text { Promedio } h t * \\
\text { (rango) }\end{array}$} \\
\hline & & $\begin{array}{l}\text { Pre- } \\
\text { tratamiento }\end{array}$ & $\begin{array}{c}\text { Post- } \\
\text { tratamiento }\end{array}$ & $\begin{array}{c}\text { Fórmula } \\
1\end{array}$ & $\begin{array}{c}\text { Fórmula } \\
2\end{array}$ & $\begin{array}{c}\text { Fórmula } \\
3\end{array}$ & $\begin{array}{c}\text { Pre- } \\
\text { tratamiento }\end{array}$ & $\begin{array}{c}\text { Post- } \\
\text { tratamiento }\end{array}$ \\
\hline A & 21 & $\begin{array}{c}840 \\
(420-600)\end{array}$ & $0,95(0-20)$ & 99,8 & 99,9 & 99,8 & $\begin{array}{c}\text { No } \\
\text { corresponde }\end{array}$ & $\begin{array}{c}\text { No } \\
\text { corresponde }\end{array}$ \\
\hline B & 17 & $\begin{array}{c}1253 \\
(400-4.800) \\
\end{array}$ & $\begin{array}{c}1,76 \\
(0-30) \\
\end{array}$ & 99,8 & 99,8 & 99,8 & $21(15-29)^{a}$ & $28(19-31)^{b}$ \\
\hline C & 14 & $\begin{array}{c}833(300- \\
1.290)\end{array}$ & 0 & 100 & 100 & 100 & $24(21-30)^{a}$ & $25(20-31)^{a}$ \\
\hline
\end{tabular}

* letras diferentes en la misma fila, difieren estadísticamente $p<0,05$ (test de Student, InfoStat Universidad Nacional de Córdoba, wwwlnfoStat.com.ar )

experiencia B sobre la C podría ser atribuible a la mayor presencia del género Haemonchus (caracterizado por su hematofagia) parasitando a estos animales.

A pesar del desarrollo de la resistencia y de los fuertes cuestionamientos a la sustentabilidad del control químico, los antihelmínticos son actualmente insumos indispensables en la producción caprina bajo condiciones pastoriles. De acuerdo a los resultados del presente estudio, el monepantel presenta un interesante potencial de uso, pero debería considerarse que la droga no está registrada en el SENASA para su empleo en caprinos. El uso fuera de marbete constituye una situación excepcional y es potestad exclusiva del médico veterinario, quien debería evaluar cuidadosamente esta situación antes de indicar su empleo. En este contexto, debería considerarse también que el monepantel no debe ser administrado a ovinos y caprinos cuya leche es destinada al consumo humano (EMA, 2012). Bajo las consideraciones citadas, la droga podría ser de utilidad en tratamientos selectivos de cuarentena, por ejemplo al ingreso de reproductores provenientes de áreas o establecimientos donde son comunes nematodes con resistencia múltiple o en aquellos hatos donde debido a estos fenómenos, ya no existan otros antihelmínticos eficaces. Luego de su administración oral, el monepantel se transforma en el metabolito activo monepantel sulfona (Sager et al., 2009), y la vida media de esta última es menor en cabras que en ovejas, probablemente por un mayor "clearance" en los caprinos (Rolfe et al., 2011). Así mismo, la droga necesita siempre ser utilizada en rotación con otros antihelmínticos como ha sido recomendado enfáticamente por Kaminsky et al. (2013) a los fines de evitar el desarrollo rápido de resistencia como ya ha ocurrido recientemente en ovinos de Uruguay, Holanda, Australia y Brasil (Mederos et al., 2014; Van der Brom et al., 2015; Sales \&Love, 2015; Cintra et al., 2016) e inclusive en caprinos de Nueva Zelanda (Scott et al., 2013).

\section{Agradecimientos}

Al convenio INTA-AUDEAS-CONADEV (CIAC 940143), al PE INTA (1115055) y a la secretaría investigación de la Universidad Católica de Córdoba por la financiación del presente trabajo.

\section{Bibliografía}

Aguirre D, Cafrune MM. 2013. Epidemiología e impacto productivo de nematodos en la región del NOA. En: Enfermedades Parasitarias de Importancia Clínica y Productiva en Rumiantes. Fundamentos epidemiológicos para su prevención y control. Fiel C. y Nari A. Editorial Hemisferio Sur, SRL (Uruguay), 115-129.

Anziani OS \& Fiel CA. 2015. Resistencia a los anti-helmínticos em nematodos que parasitan a los rumiantes en la Argentina. RIA 41: 34-46

Anziani O, Caffe G, Cooper L, Caparros J, Mohn, C, Aguilar S. 2010. Parásitos internos y caprinos de leche. Parte 2. Estudios sobre la resistencia de los nematodes gastrointestinales a los antihelmínticos. Resultados de Investigación Lechera. Proyecto Lechero. Ficha Técnica no 15 www.inta.gov.ar/lechería

Anziani OS \& Muchiut S. 2014. Resistencia antihelmíntica múltiple (closantel, febendazole, ivermectina y levamisol) en Haemonchus spp. parasitando a ovinos en la provincia de Santa Fe. Ineficacia de una triple combinación de estas drogas para su control. Rev. Med. Vet. (Buenos Aires, Argentina) 95: 22-27.

Besier B. 2007. New anthelmintics for livestock: the time is right. Trends Parasitol. 23: 21-24. 
Cabaret J, Berrag B. 2004. Faecal egg count reduction test for assessing anthelmintic efficacy: average versus individually based estimations. Vet. Parasitol.121: 105113.

Cintra MCR, Teixeira VN, Nascimento LV, Sotomaior CS. 2016. Lack of efficacy of monepantel against Trichostrongylus colubriformis in sheep in Brazil. Vet. Parasitol. 216: 4-6.

Coles GC, Bauer C, Borgsteede FH, Geerts S, Klei TR, Taylor MA, Waller PJ. 1992. World Association for the Advancement of Veterinary Parasitology (W.A.A.V.P.) methods for the detection of anthelmintic resistance in nematodes of veterinary importance. Vet. Parasitol. 44: $35-44$.

Dobson RJ, Hosking BC, Jacobson CL, Cotter JL, Besier RB, Stein PA, Reid SA. 2012. Preserving new anthelmintics: A simple method for estimating faecal egg count reduction test (FECRT) confidence limits when efficacy and/or nematode aggregation is high. Vet. Parasitol. 186:79-92.

EMA (European Medicines Agency). 2012. Monepantel (caprine and ovine species). Committee for Medicinal Products for Veterinary Use. European Public MRL assessment report (EPMR). $14 \mathrm{pp}$.

Falzon LC, van Leeuwen J, Menzies PI, Jones-Bitton A, Sears W, Jansen JT, Peregrine AS. 2015. Comparison of calculation methods used for the determination of anthelmintic resistance in sheep in a temperate continental climate. Parasitol Res.114: 1631-16434.

Hoste H., Sotirakis S., Landaus Y., Jackson F., Beveridge I. 2010. Goat -nematode interactions: think differently. Trends Parasitol. 26: 376-381.

Kaminsky R, Ducray P, Jung M, Clover R, Rufener L, Bouvier J, Weber SS, Wenger A, Wieland-Berghausen $S$, Goebel T, Gauvry N, Pautrat F, Skripsky T, Froelich O, KomoinOka C, Westlund B, Sluder A, Mäser P. 2008. A new class of anthelmintics effective against drug-resistant nematodes. Nature 452:176-80.

Kaminsky R, Rufener L, Bouvier J, Lizundia R, Schorderet Weber S,Sager H. 2013. Worms-A "license to kill". Vet. Parasitol. 195:286-291.

Kaplan RM. 2004. Drug resistance in nematodes of veterinary importance: a status report. Trends Parasitol. 20:477481.

Lespine A , Chartier C, Hoste H, Alvinerie M. 2012. Endectocides in goats: Pharmacology, efficacy and use conditions in the context of anthelmintics resistance. Small Rum. Res. 103: 10-17

McKenna PB. 2006. Further comparison of faecal egg count reduction test procedures: Sensitivity and specificity. N. Z. Vet. J. 54:365-366

Mederos AE, Ramos Z, Banchero GE. 2014. First report of monepantel Haemonchus contortus resistance on sheep farms in Uruguay. Parasit. Vectors 7:598.

Roberts F \& O'Sullivan P. 1949. Methods for egg counts and larval culture for strongyles infesting gastrointestinal tract of cattle. Aust. J. Agric. Res. 1: 99-102.

Rolfe P, Gager H, Schmidt V, Browing A, Allen B, VanHoff K, Miller S, George A, Nottingham R, Potgieter N. 2011. Efficacy and pharmacokinetics of Zolvix (monepantel) against gastrointrestinal parasites in goats. Proceedings 23rd International Conference of the World Association for the Advancement of Veterinary Parasitology. Session A, 46

Romero JR, Sánchez R, Boero C. 2007. Nematodes. Epidemiología y control. Epidemiología de la gastroenteritis verminosa de los ovinos en la pampa húmeda y la mesopotámica. En: Enfermedades parasitarias de los ovinos y otros rumiantes menores en el cono sur de América. Suárez $V$, Olaechea $F$, Romero J y Rossanigo C. INTA. Publicación Técnica N.o 70, ISSN 0325-2132, pp 33-42.

Romero JR, Anziani OS, Cetra B, Fiel CA. 2013. Epidemiologia e impacto productivo de nematodes gastrointestinales en la región NEA. En: Enfermedades Parasitarias de Importancia Clínica y Productiva en Rumiantes. Fundamentos epidemiológicos para su diagnóstico y control. Fiel C y Nari A. Ed: Agropecuaria Hemisferio Sur SRL. Uruguay. pp 89-112.

Sager H, Hosking B, Bapst B, Stein P, Vanhoff K, Kaminsky R. 2009. Efficacy of the amino-acetonitrile derivative, monepantel, against experimental and natural adult stage gastro-intestinal nematode infections in sheep. Vet. Parasitol. 159: 49-54.

Sales N \& Love S. 2015. Multi-drug including monepante (Zolvix ${ }^{\circledast}$ ) resistant Haemonchus confirmed in sheep on a farm in Greater Sydney region. https://wormmailinthecloud.wordpress.com/tag/mon epantel-resistance/in-nsw-australia/

(Verificado: 23/05/2016).

Scott I, Pomroy WE, Kenyon PR, Smith G, Adlington B and Moss A. 2013. Lack of efficacy of monepantel against Teladorsagia circumcincta and Trichostrongylus colubriformis. Vet. Parasitol. 198: 166-171.

Steffan P, Sánchez E, Entrocasso C, Fiel C, Lloberás M, Riva E, Guzmán M. 2011. Eficacia de monepantel contra nematodes de ovinos con resistencia antihelmíntica múltiple en la Región Templada de Argentina. Vet. Arg. 28

Van der Brom R, Moll L, Kappert C, Vellema P. 2015. Haemonchus contortus resistance to monepantel in sheep. Vet. Parasitol. 209: 278-280.

Waller P. 2003. Global perspectives on nematode parasite control in ruminant livestock: the need to adopt alternatives to chemotherapy, with emphasis on biological control. Anim. Health. Res. Review 4: 35-43. 